\title{
Serendipitous discovery of a strong-lensed galaxy in integral field spectroscopy from MUSE
}

\author{
Lluís Galbany $^{1 \star}$, Thomas E. Collett ${ }^{2}$, Jairo Méndez-Abreu ${ }^{3,4}$, \\ Sebastián F. Sánchez ${ }^{5}$, Joseph P. Anderson ${ }^{6}$, Hanindyo Kuncarayakti ${ }^{7,8}$. \\ ${ }^{1}$ PITT PACC, Department of Physics and Astronomy, University of Pittsburgh, Pittsburgh, PA 15260, USA. \\ ${ }^{2}$ Institute of Cosmology $\&$ Gravitation, University of Portsmouth, Dennis Sciama Building, Portsmouth, PO1 3FX, UK. \\ ${ }^{3}$ Instituto de Astrofísica de Canarias, C/ Vía Láctea s/n, E-38205, La Laguna, Spain. \\ ${ }^{4}$ Departamento de Astrofísica, Universidad de La Laguna, E-38206, La Laguna, Spain. \\ ${ }^{5}$ Instituto de Astronomía, Universidad Nacional Autónoma de México, A.P. 70-264, 04510 México, D.F., Mexico. \\ ${ }^{6}$ European Southern Observatory, Alonso de Cordova 3107 Casilla 19001 - Vitacura - Santiago, Chile. \\ ${ }^{7}$ Finnish Centre for Astronomy with ESO (FINCA), University of Turku, Väisäläntie 20, 21500 Piikkiö, Finland. \\ ${ }^{8}$ Tuorla Observatory, Department of Physics and Astronomy, University of Turku, Väisäläntie 20, 21500 Piikkiö, Finland.
}

Accepted - . Received —; in original form -

\begin{abstract}
2MASX J04035024-0239275 is a bright red elliptical galaxy at redshift 0.0661 that presents two extended sources at $2^{\prime \prime}$ to the north-east and $1^{\prime \prime}$ to the south-west. The sizes and surface brightnesses of the two blue sources are consistent with a gravitationally-lensed background galaxy. In this paper we present MUSE observations of this galaxy from the All-weather MUse Supernova Integral-field Nearby Galaxies (AMUSING) survey, and report the discovery of a background lensed galaxy at redshift 0.1915 , together with other 15 background galaxies at redshifts ranging from 0.09 to 0.9 , that are not multiply imaged. We have extracted aperture spectra of the lens and all the sources and fit the stellar continuum with STARLIGHT to estimate their stellar and emission line properties. A trace of past merger and active nucleus activity is found in the lensing galaxy, while the background lensed galaxy is found to be star-forming. Modeling the lensing potential with a singular isothermal ellipsoid, we find an Einstein radius of $1{ }^{\prime \prime} 45 \pm 00^{\prime \prime} 04$, which corresponds to $1.9 \mathrm{kpc}$ at the redshift of the lens and it is much smaller than its effective radius $\left(r_{\text {eff }} \sim 9^{\prime \prime}\right)$. Comparing the Einstein mass and the STARLIGHT stellar mass within the same aperture yields a dark matter fraction of $18 \% \pm 8 \%$ within the Einstein radius. The advent of large surveys such as the Large Synoptic Survey Telescope (LSST) will discover a number of strong-lensed systems, and here we demonstrate how wide-field integral field spectroscopy offers an excellent approach to study them and to precisely model lensing effects.
\end{abstract}

Key words: techniques: spectroscopic - gravitational lensing: strong - galaxies: general - galaxies: high-redshift

\section{INTRODUCTION}

General relativity predicts that light from distant galaxies is deflected by foreground massive objects, such as more nearby galaxies or clusters. In cases where the source and deflector are sufficiently well aligned, multiple images of the source will form around the lensing foreground object (Einstein 1915; Zwicky 1937).

Strong gravitational lensing produces highly magnified images that allow the study of fainter and smaller galax-

* E-mail: llgalbany@pitt.edu (LG) ies than would otherwise be possible with current instrumentation (Narayan \& Bartelmann 1996). Also, it provides the most precise and accurate method to determine the masses of galaxies in a model-free fashion being independent of assumptions such as the initial mass function (IMF) or galaxy dynamics (Treu 2010). In addition, the comparison between the inferred total mass distribution in strong lenses to their observed brightness profile provides valuable information about the galaxy dark matter content and distribution (Bertin et al. 1994; Cappellari et al. 2015). Strong lensing systems can also place constraints on cosmological parameters (Bonvin et al. 2017; Collett \& Auger 2014). 
Low redshift lenses are particularly interesting systems as their Einstein radii form at a much smaller physical scale than for higher redshift lenses. This enables studies of the central matter distribution in these lenses. Their much lower distances also allow more detailed follow-up of the lens kinematics and stellar populations. Recent studies have combined these approaches to place tight constraints on the IMF (Smith et al. 2015; Newman et al. 2017), the validity of General relativity (Collett 2018a), and the Hubble constant (Collett et al 2018b, in prep). Unfortunately, despite the utility of low redshift strong lenses only a handful are known (Smith et al. 2005, 2015) due to the rarity of massive galaxies and the small volume at very low redshifts.

While some lensed systems have been discovered serendipitously by visually checking images of the lens, there have been several dedicated attempts to identify strong gravitational lensed objects around massive galaxies. Previous search strategies typically used both photometric or spectroscopic methods. Photometric methods include searching for arc morphologies (e.g. Blakeslee et al. 2004), or outliers in color diagrams (e.g. Gavazzi et al. 2014), given that foreground and background objects have different colors. The majority of these methods are based on a priori knowledge of galaxy properties, and visual inspection is usually required. Most recently, new automatic methods based on deep learning are flowering (Lanusse et al. 2018). Alternatively, spectroscopic methods that are based on detecting rogue emission (or absorption) lines in galaxy spectra (Bolton et al. 2006) have been employed.

Recently, Smith (2017) reported the discovery of a lensed galaxy in integral field spectroscopy (IFS) observations of SDSS J170124.01+372258.0 from the MaNGA survey (Bundy et al. 2015) This methodology has been greatly extended by Talbot et al. (2018) to discover 38 lenses from MaNGA, with lens redshifts spanning $0.02<z_{l}<0.13$. Despite the clear detection of weak emission lines in the datacube corresponding to a star-forming background galaxy at different redshift than the lens, the low spatial resolution of MaNGA data does not yet allow for a detailed understanding of the observed lensing mass distribution.

We here report the discovery of strong gravitational lensing by the galaxy 2MASX J04035024-0239275 ${ }^{1}$, in IFS obtained with the Multi Unit Spectroscopic Explorer (MUSE) on the Very Large Telescope (VLT), an IFU instrument with high resolutions in both spectral $(R \sim 3000)$ and spatial $\left(0.2^{\prime \prime} /\right.$ spaxel $)$ dimensions, which permits a detailed reconstruction of the source.

In section 2 we describe the observations. Then, in section 3 we describe the lens model and the residual datacube after subtraction. In section 4 the lensed source. In Section 5 we present a simple model of the observed image configuration and the lensing mass. In section 7 we summarize and list our conclusions. Throughout this work we use the best fit cosmological parameters of Planck Collaboration et al. (2016).

1 We note that this lens has also been independently discovered by Collier et al. (2018), using the same MUSE data from our ESO programme 098.D-0115(A); PI: Galbany.
Table 1. Coordinates and redshifts of all galaxies found in the FoV, including the lens galaxy $(A)$, the lensed source $(B+C)$, and the merger $(\mathrm{S})$.

\begin{tabular}{|c|c|c|c|}
\hline Label & R.A. & Dec & $z$ \\
\hline A (lens) & $4^{\mathrm{h}} 03^{\mathrm{m}} 50.24$ & $-02^{\circ} 39^{\prime} 27^{\prime \prime} .4$ & 0.0661 \\
\hline $\mathrm{B}\left(\right.$ Image $\left.\mathrm{I}_{+}\right)$ & $4^{\mathrm{h}} 03^{\mathrm{m}} 50.16$ & $-02^{\circ} 39^{\prime} 29^{\prime \prime} 2$ & 0.1915 \\
\hline C (Image $\left.I_{-}\right)$ & $4^{\mathrm{h}} 03^{\mathrm{m}} 50.25$ & $-02^{\circ} 39^{\prime} 26^{\prime \prime} 6$ & 0.1915 \\
\hline $\mathrm{D}$ & $4^{\mathrm{h}} 03^{\mathrm{m}} 50.79$ & $-02^{\circ} 39^{\prime} 40^{\prime \prime} 2$ & 0.4556 \\
\hline $\mathrm{E}$ & $4^{\mathrm{h}} 03^{\mathrm{m}} 50.53$ & $-02^{\circ} 39^{\prime} 42^{\prime \prime} ._{0}$ & 0.4551 \\
\hline $\mathrm{F}$ & $4^{\mathrm{h}} 03^{\mathrm{m}} 49.71$ & $-02^{\circ} 39^{\prime} 39^{\prime \prime} .^{6}$ & 0.4538 \\
\hline $\mathrm{G}$ & $4^{\mathrm{h}} 03^{\mathrm{m}} 49.49$ & $-02^{\circ} 39^{\prime} 28^{\prime \prime} .2$ & 0.4564 \\
\hline $\mathrm{H}$ & $4^{\mathrm{h}} 03^{\mathrm{m}} 48.74$ & $-02^{\circ} 39^{\prime} 31^{\prime \prime} .2$ & 0.4542 \\
\hline $\mathrm{I}$ & $4^{\mathrm{h}} 03^{\mathrm{m}} 49.30$ & $-02^{\circ} 39^{\prime} 23^{\prime \prime} .^{\prime}$ & 0.4549 \\
\hline $\mathrm{J}$ & $4^{\mathrm{h}} 03^{\mathrm{m}} 49.79$ & $-02^{\circ} 39^{\prime} 06^{\prime \prime}{ }^{\prime} 3$ & 0.4523 \\
\hline $\mathrm{K}$ & $4^{\mathrm{h}} 03^{\mathrm{m}} 49.59$ & $-02^{\circ} 39^{\prime} 10^{\prime \prime} 6$ & 0.0946 \\
\hline $\mathrm{L}$ & $4^{\mathrm{h}} 03^{\mathrm{m}} 49.77$ & $-02^{\circ} 39^{\prime} 03^{\prime \prime} .^{\prime} 8$ & 0.0946 \\
\hline M & $4^{\mathrm{h}} 03^{\mathrm{m}} 52^{\mathrm{s}} .02$ & $-02^{\circ} 39^{\prime} 05^{\prime \prime} .^{\prime}$ & 0.5944 \\
\hline $\mathrm{N}$ & $4^{\mathrm{h}} 03^{\mathrm{m}} 50.69$ & $-02^{\circ} 39^{\prime} 21^{\prime \prime} .^{0}$ & 0.5929 \\
\hline $\mathrm{O}$ & $4^{\mathrm{h}} 03^{\mathrm{m}} 50.27$ & $-02^{\circ} 39^{\prime} 51^{\prime \prime} 6$ & 0.5937 \\
\hline $\mathrm{P}$ & $4^{\mathrm{h}} 03^{\mathrm{m}} 50.51$ & $-02^{\circ} 39^{\prime} 48^{\prime \prime} .^{\prime} 4$ & 0.6286 \\
\hline Q & $4^{\mathrm{h}} 03^{\mathrm{m}} 51^{\mathrm{s}} .69$ & $-02^{\circ} 39^{\prime} 06^{\prime \prime} \cdot 2$ & 0.9031 \\
\hline $\mathrm{R}$ & $4^{\mathrm{h}} 03^{\mathrm{m}} 51^{\mathrm{s}} 45$ & $-02^{\circ} 39^{\prime} 07^{\prime \prime} .0$ & unkn. \\
\hline $\mathrm{S}$ (merger) & $4^{\mathrm{h}} 03^{\mathrm{m}} 50.67$ & $-02^{\circ} 39^{\prime} 30^{\prime \prime} 0$ & 0.0661 \\
\hline
\end{tabular}

\section{OBSERVATIONS}

2MASX J04035024-0239275 is a bright red elliptical galaxy at R.A. $=04^{\mathrm{h}} 03^{\mathrm{m}} 50$ s. 26 , Dec $=-02^{\circ} 39^{\prime} 27^{\prime \prime}$. 64 with a radial velocity $c z \sim 19,952 \mathrm{~km} \mathrm{~s}^{-1}$ (Jones et al. 2009), corresponding to an angular scale of $1.256 \mathrm{kpc} \operatorname{arcsec}^{-1}$. The type Ia supernova LSQ13cwp exploded 9.6" $\mathrm{N}$ and 6.1" E from the galaxy core and was discovered on November 8th 2013 (Walker et al. 2013). For this reason, 2MASX J04035024-0239275 was included in the sample of supernova host galaxies of the All-weather MUse Supernova Integral-field Nearby Galaxies (AMUSING; Galbany et al. 2016a) survey. AMUSING is an ongoing project aimed at studying the environments of supernovae ( $\mathrm{SNe}$ ) by means of the analysis of a large number of nearby SN host galaxies (redshifts typically between 0.005 and 0.1). The AMUSING sample currently comprises more than 300 galaxies observed in the last 6 semesters (P95 to $\mathrm{P} 100$ ) and is composed by a wide variety of galaxy types with the common characteristics of having hosted a known SN.

2MASX J04035024-0239275 was observed using the integral-field spectrograph MUSE (Bacon et al. 2010) at the ESO Very Large Telescope (VLT) UT4 of the Cerro Paranal Observatory during a clear night on 2016 November 7 th. In the Wide Field Mode, MUSE covers a continuous sky region of $1^{\prime} \times 1^{\prime}$ with a small spaxel size of $0.2^{\prime \prime} \times 0.2^{\prime \prime}$, which limits the spatial resolution of the data to the atmospheric seeing during the observation $\left(\sim 0.7^{\prime \prime}\right)$. MUSE observations cover the wavelength range spanning from $4750 \AA$ to $9300 \AA$, with a spectral sampling of $1.25 \AA$ and a resolving power $R$ increasing from 1800 in the blue edge to 3600 in the red edge of the spectrum. With its excellent total throughput, MUSE offers an unprecedented combination of sensitivity, spatial resolution, and field of view (FoV).

We obtained four dithered (by a few arcsec) exposures of 700 s integration each, totaling 2800 s on source, that re- 


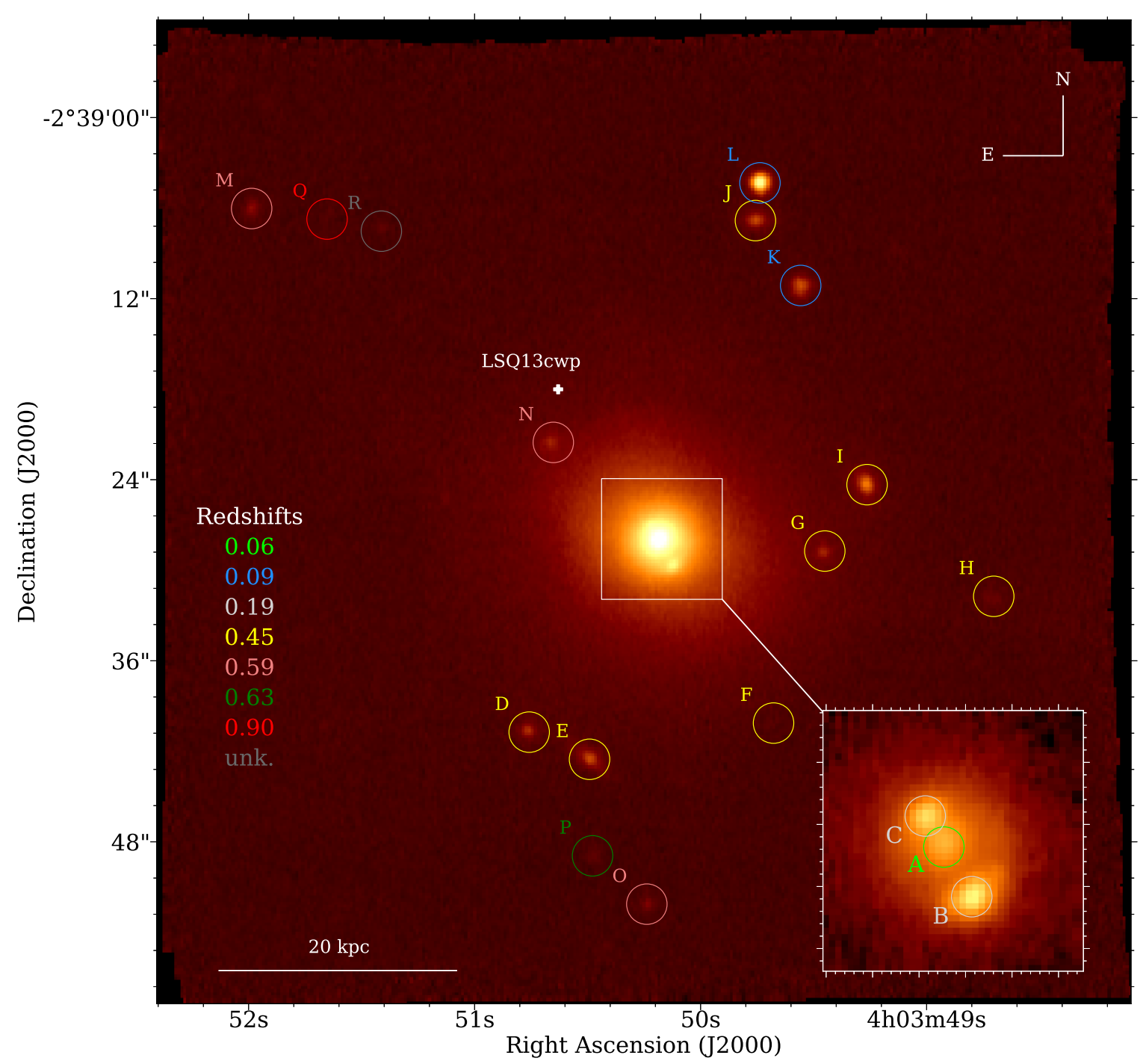

Figure 1. Synthetic $10 \AA$ narrow-band image (centered at $7184 \AA$ ) of the MUSE observation. We identified 16 different sources in the field of view, indicated with circles. The inset is a synthetic narrow-band image centered at the $\mathrm{H} \alpha$ emission of the source $(z=0.1915)$ where the lens and the two images of the source can clearly be distinguished. Supernova LSQ13cwp position is represented by a white plus sign.

sulted in a combined datacube with more than 105,000 individual spectra. There are no stars in the field of our MUSE observations, but we checked the image quality of the slowguiding system (SGS) that uses metrology fields around the MUSE field of view (FoV) to provide a secondary telescope guiding. Three stars were detected in those fields with an average FWHM of $0.676 \pm 0.040$ arcsec. We therefore assume throughtout the paper the point spread function (PSF) is a circular Gaussian with a full-width half-maximum of $0.7^{\prime \prime}$, which corresponds to a physical resolution of $880 \mathrm{pc}$ at the redshift of the lens, and it is consistent with the reported DIMM seeing. Data reduction was performed following prescriptions reported in Galbany et al. (2016a) and Krühler et al. (2017), and the reader is referred to those papers for details.

IFS can be thought as a sequence of two-dimensional (2D) very narrow-band imaging of the FoV covered by the instrument. In the case of MUSE, this translates into 

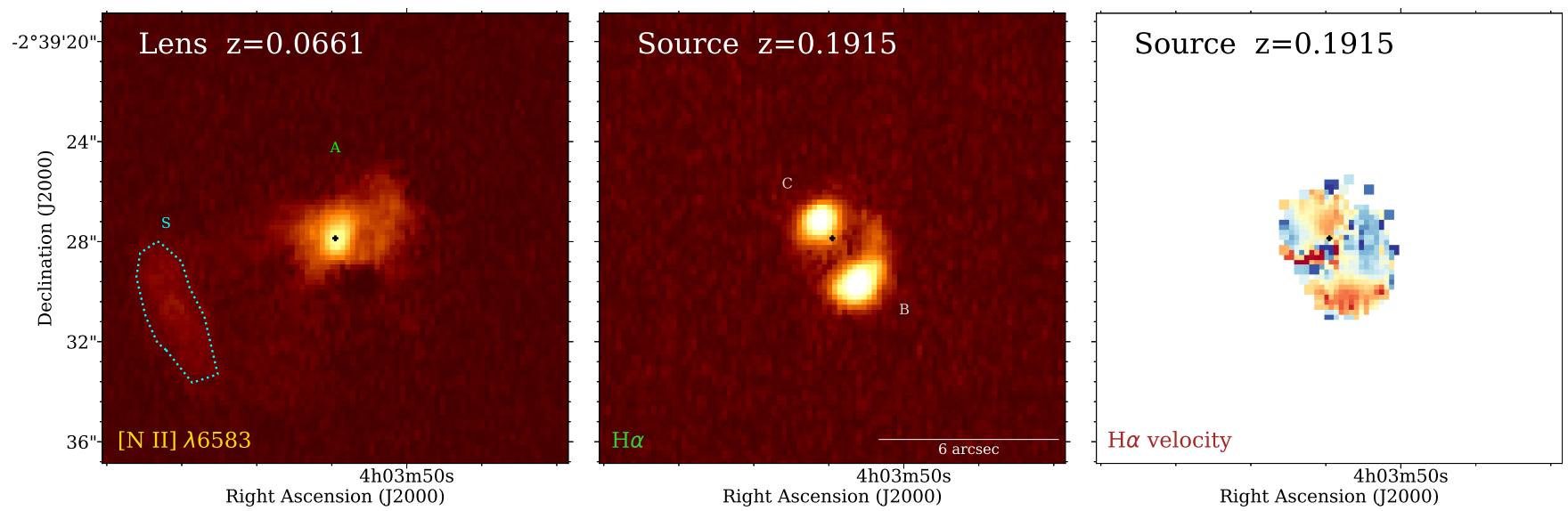

Figure 2. $22^{\prime \prime} \times 22^{\prime \prime}$ cutouts centered at the core of the foreground galaxy acting as a lens. $S$ region indicates the merger region (see section 3.3). In the left panel, a [N II] $\lambda 6583$ emission line flux map of the lens galaxy (7019 $\AA$ observer-frame), and in the center and right panels, $\mathrm{H} \alpha$ flux and velocity maps of the background source galaxy (7820 $\AA$ observer-frame). An arc, forming a partial Einstein ring can clearly be identified in central and right panels.

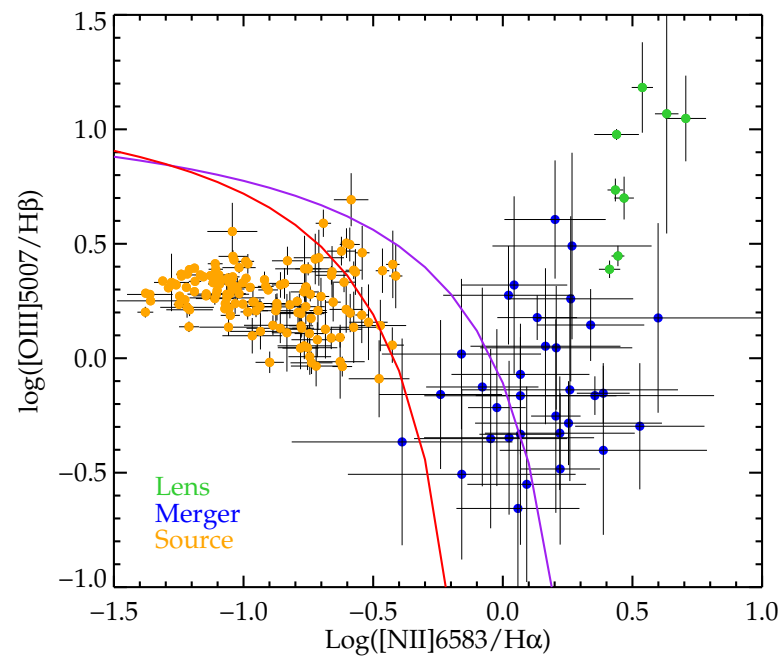

Figure 3. BPT diagram constructed from line emission ratios measured in three distinct regions: the lens (A), the lensed source (B and $\mathrm{C}$ ), and the possible merger (S). This diagram clearly shows that the origin of the ionization is different for the three objects. Purple and red solid lines correspond to the two criteria commonly used to separate star-forming (SF) from AGNdominated galaxies from Kewley et al. (2001) and Kauffmann et al. (2003), respectively.

$1.25 \AA$ wide 3,681 images. Each of these images correspond to a different rest-frame wavelength for objects at different redshifts in the FoV.

Visual inspection of the 2MASX J04035024-0239275 datacube revealed a bright detached source located a few spaxels South-West of the core of the main galaxy. A spectrum extracted with a small aperture at the position of the source resembled that of a typical star-forming galaxy at a higher redshift than the main galaxy $(z \sim 0.19)$. Examining the wavelength slice (narrow-band image) of the datacube corresponding to the $\mathrm{H} \alpha$ emission of this source $(7820 \AA)$ we discovered another source at the North-Eastern side of the 2MASX J04035024-0239275 core (see inset of Fig. 1). The spectrum of this 2 nd source was found to be very similar to the S-E object, and the estimated redshifts were consistent Further inspection of the datacube revealed up to 15 other galaxies present in the FoV. Figure 1 shows a synthetic $10 \AA$ narrow-band image from the datacube centered at $7184 \AA$ in the observer frame ${ }^{2}$, where all objects detected are marked with circles of different colors grouped by their corresponding measured redshifts. Table 1 lists coordinates and spectroscopic redshifts of all objects detected in the FoV. The inner panel presents a zoom-in of the 2MASX J04035024-0239275 core, from a synthetic narrow-band image centered at $7820 \AA$, where it can be clearly seen the core (labeled as $A$ throughout the paper) and the two S-W (labeled as $B$ ) and N-E (labeled as $C$ ) bright blobs.

The geometrical configuration of $B$ and $C$ together with their similar spectra, suggested that they may be in fact the same object lensed by 2MASX J04035024-0239275. In the rest of the paper we present an analysis of the lens $A$ and the galaxy $B+C$, and demonstrate that this is actually the case.

\section{THE LENS}

\subsection{Reconstructing the lens}

Although the images of the background galaxy are clearly seen in the observed cube, their visibility might be enhanced by subtracting a model of the contribution of the foreground galaxy acting as a gravitational lens. We have explored a number of approaches to model the spectrophotometric properties of the lens, namely: isophotal fitting, analytical Sérsic fitting, and single stellar population (SSP) synthesis.

2 This wavelength was selected because it corresponds to the $\mathrm{H} \alpha$ emission of galaxies $L$ and $K$. They only show a few bright emission lines on top of a very faint and noisy continuum (See Figure 8 ), and otherwise they would not be visible in the image. 
Table 2. Extinction corrected flux of the most prominent gas phase emission lines and properties extracted from integrating the model datacube (for $A$ ), from aperture spectra in the residual cube after subtracting the lens model (for $B$ and $C$ ), and integrating the region defined by the dotted blue region area in Figure 2 (for $S$ ). All fluxes in units of $10^{-18} \mathrm{erg} \mathrm{s}^{-1} \mathrm{~cm}^{-2} \AA^{-1}$.

\begin{tabular}{lcccc}
\hline \hline & $\mathrm{A}($ lens $)$ & $\mathrm{B}\left(\right.$ Image $\left.\mathrm{I}_{+}\right)$ & $\mathrm{C}$ (Image $\left.\mathrm{I}_{-}\right)$ & $\mathrm{S}($ merger $)$ \\
\hline$[\mathrm{O} \mathrm{II}] \lambda \lambda 3727,29$ & - & - & - & - \\
$\mathrm{H} \delta$ & - & $408.48(28.14)$ & $180.00(27.59)$ & - \\
$\mathrm{H} \gamma$ & - & $771.80(60.97)$ & $442.67(39.02)$ & - \\
$\mathrm{H} \beta$ & $171.80(26.90)$ & $1538.85(47.76)$ & $879.77(77.80)$ & $83.05(12.44)$ \\
{$[\mathrm{O} \mathrm{III}] \lambda 4959$} & $78.19(12.24)$ & $1019.50(64.33)$ & $523.89(43.61)$ & $31.25(7.46)$ \\
{$[\mathrm{O} \mathrm{III}] \lambda 5007$} & $213.29(33.39)$ & $2930.72(92.57)$ & $1705.22(44.40)$ & $95.85(31.05)$ \\
{$[\mathrm{N} \mathrm{II}] \lambda 6548$} & $159.63(24.99)$ & $467.93(43.41)$ & $284.88(25.78)$ & $116.74(34.98)$ \\
$\mathrm{H} \alpha$ & $369.65(57.88)$ & $4401.12(121.20)$ & $2516.15(71.69)$ & $237.52(57.22)$ \\
{$[\mathrm{N} \mathrm{II}] \lambda 6583$} & $452.30(70.82)$ & $1761.82(66.67)$ & $1025.20(34.71)$ & $353.33(82.88)$ \\
{$[\mathrm{S} \mathrm{II}] \lambda 6717$} & $182.16(28.52)$ & $781.52(97.46)$ & $472.91(50.56)$ & $117.79(29.73)$ \\
{$[\mathrm{S} \mathrm{II}] \lambda 6731$} & $116.31(18.21)$ & $713.79(80.35)$ & $445.37(53.56)$ & $81.26(20.51)$ \\
\hline $\mathrm{A}_{V}^{\operatorname{gas}}[\mathrm{mag}]$ & $0.00(0.02)$ & $1.07(0.03)$ & $1.17(0.04)$ & $0.47(0.03)$ \\
$12+\log _{10}(\mathrm{O} / \mathrm{H})[\mathrm{dex}]$ & - & $8.39(0.09)$ & $8.39(0.10)$ & - \\
$\log _{10}(\mathrm{SFR}[\mathrm{M} \odot \mathrm{yr}-1])[\mathrm{dex}]$ & - & 4.261143 & 2.436128 & - \\
$\log _{10}\left(\mathrm{M}\left[\mathrm{M}_{\odot}\right]\right)[\mathrm{dex}]$ & 11.54 & 10.35 & 10.13 & 9.79 \\
$\mathrm{~A}_{V}^{*}[\mathrm{mag}]$ & 0.24 & 0.27 & 0.02 & 0.17 \\
$<\log _{10}\left(\mathrm{t}_{*}[\mathrm{yr}]\right)>[\mathrm{dex}]$ & $9.92(0.74)$ & $7.31(0.74)$ & $7.04(0.66)$ & $10.07(0.18)$ \\
$<\log _{10} \mathrm{Z}_{*}>[\mathrm{dex}]$ & $0.022(0.016)$ & $0.017(0.013)$ & $0.012(0.014)$ & $0.016(0.014)$ \\
\hline
\end{tabular}

STARLIGHT best fits are then put together in a $3 \mathrm{D}$ datacube that describes a model of the lens galaxy.

\subsection{Separating the background source from the lens}

In order to separately study the lens and the background source, we removed the contribution of the lens from the source by subtracting the SSP fitting datacube from the $3 \mathrm{D}$ observation. The residual contains the ionized emission lines from the lens (consistent with a redshift 0.0661) on top of both the stellar and the gas-phase emission of the source (at redshift 0.1915). We fit all strong emission lines of ionized gas from the lens and the background source $(\mathrm{H} \beta$, [O III] $\lambda 5007,[\mathrm{~N} \mathrm{II}] \lambda 6548, \mathrm{H} \alpha,[\mathrm{N}$ II] $\lambda 6583)$ using PIPE3D (Sánchez et al. 2016).

For the lens, the $[\mathrm{N} \mathrm{II}] \lambda 6583$ emission is stronger than $\mathrm{H} \alpha$ suggesting that the underlying ionization is caused by an AGN. In Figure 2 (left panel) we show the [NII] $\lambda 6583$ $2 \mathrm{D}$ emission line map of the lens, where the two blobs from the background galaxy are not present. The AGN origin is confirmed by the location of the emission line ratios in the AGN region on the BPT diagram shown in Figure 3.

On the other hand, the lensed source object shows strong $\mathrm{H} \alpha$ emission indicating recent star-formation. In the central panel of Figure 2 we show the $\mathrm{H} \alpha$ 2D emission line map, where the two images and a ring structure can clearly be seen. We note that the region within the images of the source appear clean of emission from the lens, confirming that the SSP approach works better in the lens core region. Similarly, the origin of the ionization as star-forming activity is confirmed by the location of the flux ratios in the BPT diagram (See Figure 3).

By determining the central wavelength of the $\mathrm{H} \alpha$ emission of the source in each individual spaxel, we can reconstruct the velocity field of the two images. In the right panel of Figure 2 we show how the projected rotational velocity along the line-of-sight is in the opposite direction in each of the images. We found velocities in the range $v \in-50,50 \mathrm{~km}$ $\mathrm{s}^{-1}$.

\subsection{Possible merger remnant}

Another interesting result from our SSP fitting that is also evident in the left panel of Figure 2, is the presence of a residual shell-like structure on the eastern side of the lens (labeled as $S$ hereafter). From inspection of the original cube, this structure is clearly at the same redshift of the lens, although it presents emission lines from ionized gas. The integrated spectra of the lens model and that corresponding to the source encircled by the dotted blue region area represented in Figure 2, are shown in black in Figure 4. They both have red stellar continua characteristic of old stellar populations with faint gas emission lines lying on top. The stellar continuum is noisier than the lens core but we still get a meaningful SSP fit from STARLIGHT that is shown in red. The two inner panels zoom-in to the wavelengths around $\mathrm{H} \beta$ and $\mathrm{H} \alpha$. In blue we show the difference between the observation and the SSP fit, where emission lines can clearly be identified. On the right side, the reconstructed star formation history is presented, which shows that $96.25 \%$ and $99 \%$ of the SSP models needed for the best fit of the lens and the merger, respectively, correspond to populations of ages older than 3 Gyr ( $\sim 9.5$ dex). Stellar parameters from the SSP synthesis such as the average stellar age, metallicity, stellar extinction and stellar mass, as well as properties estimated from the gas such as the gas extinction, oxygen abundance and on-going star formation rate are reported in Table 2 .

The presence of ionized gas in early-type galaxies is known to be more frequent than what was considered in recent times (e.g. Sarzi et al. 2010; Papaderos et al. 2013; Singh et al. 2013). The source of the ionization is generally a 

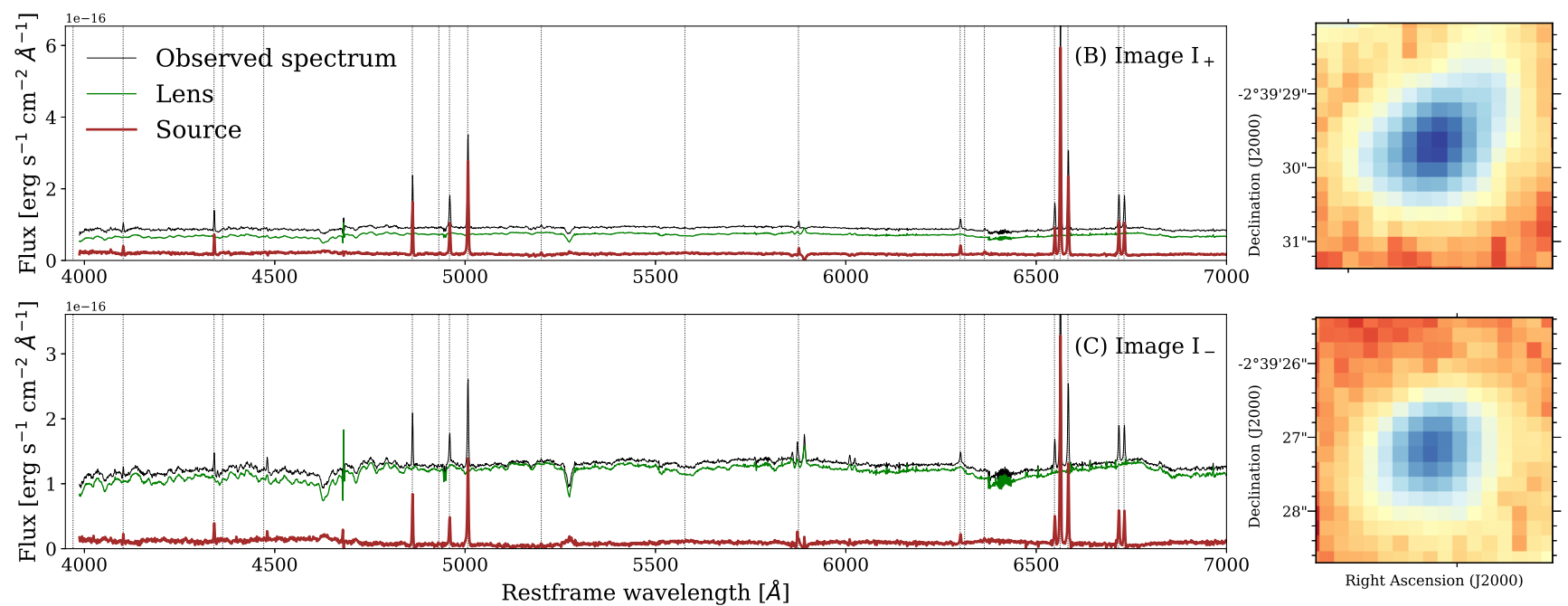

Figure 5. Spectra of images $\mathrm{I}_{+}\left(\right.$source $B$ ) and $\mathrm{I}_{-}$(source $C$ ). In black we show the observed spectrum obtained from a 2 arcsec diameter aperture, in green the model of the lens extracted from an aperture of the same size in the synthetic model datacube, and in red the residual. On the right side, the $\mathrm{H} \alpha$ emission $2 \mathrm{D}$ map of both sources.

mix between different physical processes, including four major sources: (i) nuclear activity (i.e., AGN), that have a characteristic ionization with strong $[\mathrm{N} \mathrm{II}] / \mathrm{H} \alpha$ and $[\mathrm{O}$ III $] / \mathrm{H} \beta$ ratios (e.g. Gomes et al. 2016b); (ii) outflows by sometimes undiscovered central AGNs (e.g. Kehrig et al. 2012; Cheung et al. 2016); (iii) ionization by young stars, remnants of a dimming disk or a consequence of a rejuvenation by the capture of a gas rich satellite galaxy (e.g. Gomes et al. 2016a); and, in most of the cases, (iv) diffuse ionization by postAGB stars (Binette et al. 1994; Stasińska et al. 2008; Sarzi et al. 2010; Papaderos et al. 2013; Singh et al. 2013; Gomes et al. 2016b; Belfiore et al. 2016). Although the nature of the ionization is more or less clear, it is still unclear the origin of this ionized gas. Although there are elliptical galaxies without trace of either ionized or cold gas, many of them show both phases of the gas (Sanchez et al. 2017). Thus, maybe this gas represents the left-overs of the early evolution of these galaxies, that once were gas rich, star-forming, and have halted their activity due to gas starvation (e.g. Bekki et al. 2002).

However, the detected structure, named as $S$, comprises both ionized gas and stellar populations, and it presents a shell-like structure located at the SE of the lens, with a diffuse (smoother) distribution towards the center of the galaxy. Its morphology is different from the one expected either by a direct ionization by an AGN or a centrally distributed gas, or the spiral-like structure of ionization due to young stars. Its location in the BPT diagram indicates that the source of the ionization is LINER-like, either associated with post-AGBs or shocks (See Figure 3). Even more, shell-like structures are characteristic of past merging events, that disrupted the morphology of the captured galaxy and/or created resonances in the distorted disk (e.g. García-Lorenzo et al. 2005). Altogether we consider that this structure is totally unrelated to the lensed galaxy, being the remnant of a past merging event. We consider that the lens galaxy has captured, disrupted and integrated a less massive object, more gas-rich, and it has produced the observed structure due to dynamical processes. The gas remnant is ionized either by post-AGB stars, shocks or a combination of both processes.

\section{THE BACKGROUND SOURCE}

We estimated the position of the galaxy lens core and the center of the two source images by fitting a 2D gaussian profile in the [N II] $\lambda 6583$ and the $\mathrm{H} \alpha$ emission map, respectively (See Figure 2). We obtained an angular distance from the lens core to the south-western (B) image of $\sigma_{+}=2.056$ arcsec $(1.58 \mathrm{kpc})$, and to the north-eastern image of $\sigma_{-}=-1.013$ $\operatorname{arcsec}(1.27 \mathrm{kpc})$.

To study the source we extracted $2^{\prime \prime}$-diameter aperture spectra at the position of the two $B$ and $C$ images, in both the observed datacube and in the synthetic lens model. In Figure 5 we show the observed spectra of the $B$ and $C$ images in black lines, the lens spectrum in green, and the subtraction residual in red. On the right, the $\mathrm{H} \alpha$ emission 2dimensional map of both sources is shown. STARLIGHT fit results of the residual spectra are listed in Table 2 .

Light from both images suffer different amounts of extinction while traveling through different sides of the lens. A larger amount of extinction is reflected in a higher observed $H \alpha / H \beta$ ratio, which once corrected increases the flux of $\mathrm{H} \beta$ and [O III] $\lambda 5007$ over $\mathrm{H} \alpha$ and [N II] $\lambda 6583$, but does not affect (significantly) both $[\mathrm{O} \mathrm{III}] / \mathrm{H} \beta$ and $[\mathrm{N} \mathrm{II}] / \mathrm{H} \alpha$ ratios. $A_{V}$ measured from the Balmer decrement, $\mathrm{H} \alpha$ over $\mathrm{H} \beta$ observed ratio, assuming an $R_{V}$ of 3.1, is 1.07 (0.03) mag for $B$ and 1.17 (0.04) mag for $C$. In addition, the stellar extinction obtained from the SSP fitting $A_{V}^{*}$ is 0.27 for $B$ and 0.02 for C.

Oxygen abundance is measured in the two spectra using the O3N2 calibrator from Marino et al. (2013). Interestingly, we find the same value from the spectra of two images, 8.39 dex. This is expected and confirms that the two images correspond indeed to the same source galaxy. On the other 

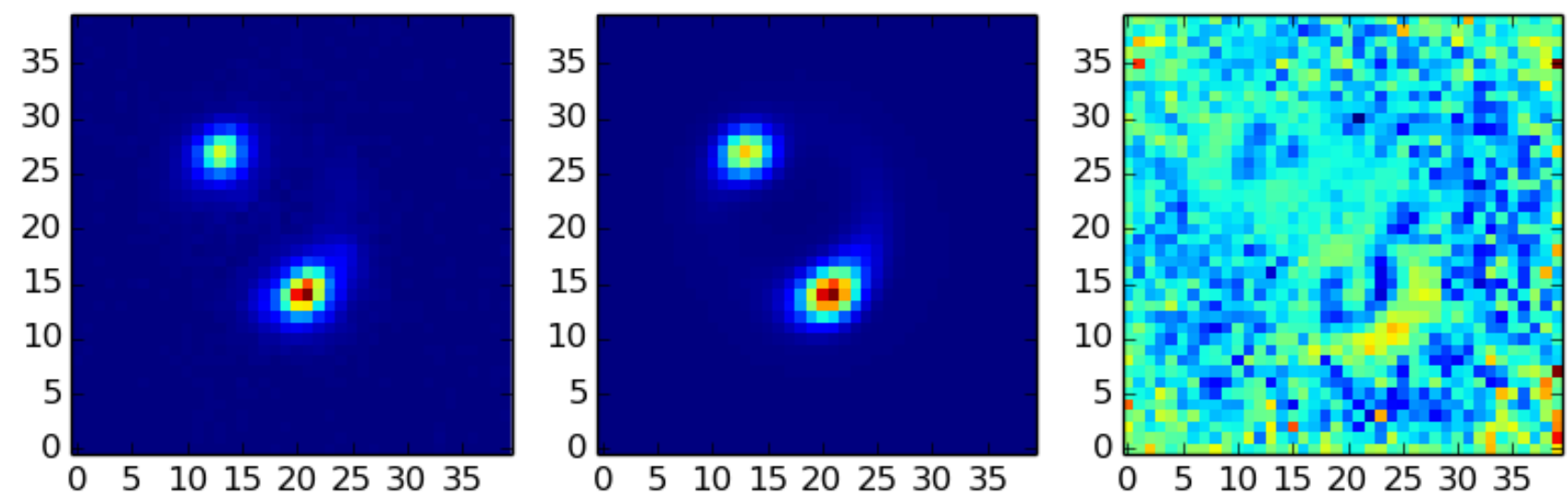

Figure 6. Best fit reconstruction of the lensed images assuming a SIE lens model and a single Sérsic profile for the source. Coordinates are in 0.2 " MUSE pixels. From left to right: data, model, residual. The residuals are poor because the PSF is not well known - there are no stars in the field of view.

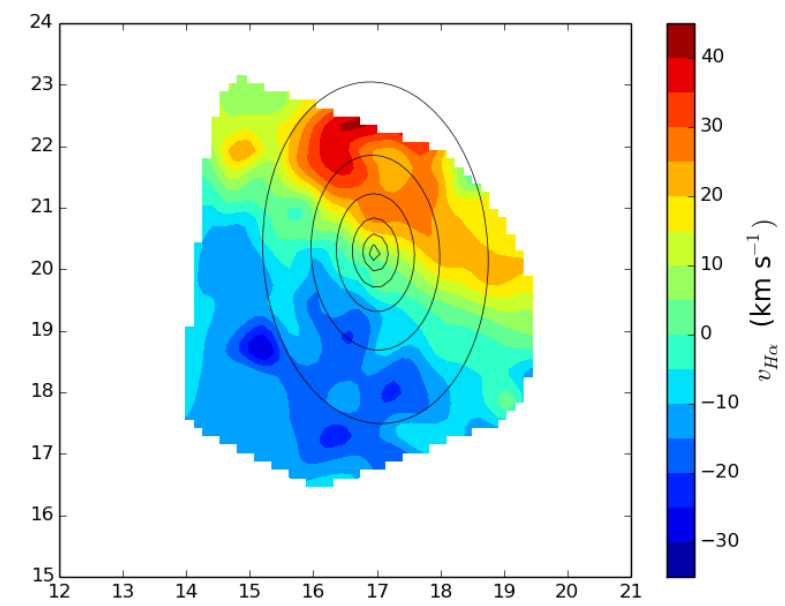

Figure 7. Velocity reconstruction of the source from tracing the observed $\mathrm{H} \alpha$ velocity onto the source plane using the best fit lens model. The best fit reconstructed light profile is indicated by the black contours.

hand, the star formation rate (SFR) estimated through the expression provided by Kennicutt (1998) using the $\mathrm{H} \alpha$ emission flux, is a factor 1.75 higher for $B$ than for $C$. We argue below that this different strength of the emission is due to the magnification effect, which is in turn different for each image.

\section{LENS MODELING}

The observed positions and fluxes of the multiple images are sensitive to the total lensing mass and its distribution within the lens. Since the observed images are extended, families of lens models that reproduce the observed image separations, but not the shape of the lensed features can be excluded.

Extracting this information requires a detailed recon- struction of the observed source light profile. By ray-tracing all of the observed image plane pixels through a lensing mass model back on the source plane to reconstruct a single selfconsistent source.

To reconstruct the observed light profile of the arcs, we fit a singular isothermal ellipsoid (SIE) for the lensing mass and a single elliptical Sérsic profile for the background source. We allow the Einstein radii, positions, position angle, and flattening of the lens to be free parameters and we do not fix any to the observed lens positions.

The model is able to reconstruct the observed light profile, including the tail of the arc to the North West of image B. The best fit model is shown in Figure 6. The Einstein radius is $1.45 \pm 0.04$ arcsec. We estimate errors from fitting with a PSF of $0.6^{\prime \prime}$ and $0.8^{\prime \prime}$ FWHM. The inferred lens center of mass and the flattening and ellipticity are consistent with the observed light profile.

The observed Einstein radius corresponds to a physical scale of $1.9 \mathrm{kpc}$ and an enclosed lensing mass of $1.08 \pm 0.06 \times$ $10^{11} M_{\odot}$.

\subsection{Dark matter fraction}

Comparing the lensing and the stellar masses allows us to estimate the dark matter mass enclosed within the Einstein radius. To measure the dark matter fraction, we extracted a circular aperture spectrum from the $3 \mathrm{D}$ datacube of the STARLIGHT model of the lens (second approach described in section 3) with an angular size of the Einstein radius $\left(1.45^{\prime \prime}\right)$, and analyzed the spectrum with STARLIGHT to estimate the stellar mass.

The best SSP synthesis provides a star formation history with an average stellar age of $2.6 \mathrm{Gyr}$ and an average metallicity of $1.1 \mathrm{Z}_{\odot}$, with two dominant bursts at $\log _{10}(\mathrm{t}$ [yr]) 9.4 and 10.1 dex. We obtained $\log _{10} \mathrm{M}_{<\theta_{E}}^{*}\left[\mathrm{M}_{\odot}\right]=$ $10.946 \pm 0.131$ dex, which corresponds to $82 \% \pm 8 \%$ of the mass enclosed within the Einstein radius, and means that dark matter contributes $18 \% \pm 8 \%$ to the total mass within 

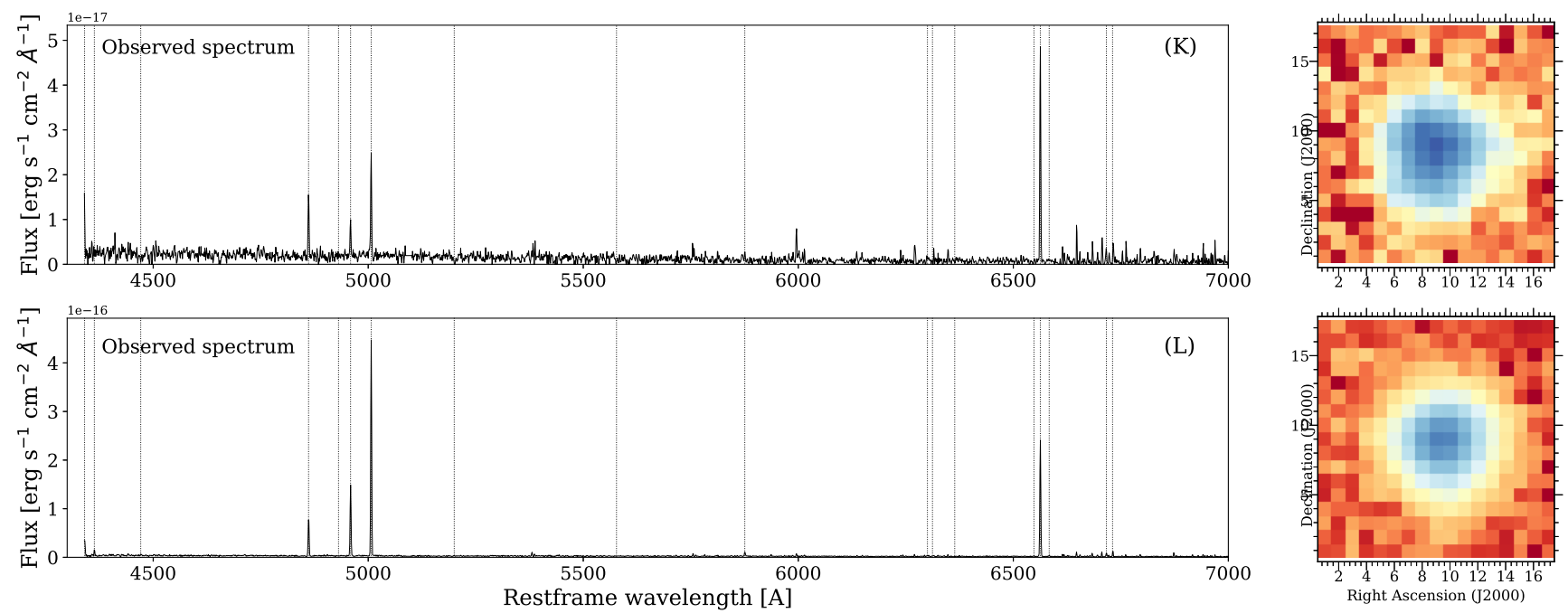

Figure 8. Spectra of background galaxies $K$ and $L$ both at $z=0.09$. They show very faint stellar continua and 4 prominent emission lines. On the right, we show cutouts of the $\mathrm{H} \alpha 2 \mathrm{D}$ emission line map.

the Einstein ring. This fraction is consistent with other reports in the literature for galaxies with similar stellar mass (Cappellari et al. 2013).

\subsection{Velocity field and dispersion}

By ray-tracing through the best fit lens model, we can reconstruct the velocity field of the source. This reconstruction is shown in Figure 7: The source shows a velocity gradient across the source, with the rotation axis offset from the inferred semi-major axis of the source. A more detailed reconstruction of both lens and source is required to investigate if this offset is real.

Assuming an isothermal lens, the Einstein radius predicts a velocity dispersion of $280 \pm 4 \mathrm{~km} \mathrm{~s}^{-1}$. Our STARLIGHT fit for the lens model within the Einstein radius has a measured velocity dispersion of $\sigma_{v}^{\mathrm{STAR}}=247 \pm$ $32 \mathrm{~km} \mathrm{~s}^{-1}$, once corrected for instrumental effects and the difference in dispersion of the bases used for the SSP synthesis. The corresponding dispersion measured in the observed spectrum within the Einstein radius is larger $298 \pm 29 \mathrm{~km}$ $\mathrm{s}^{-1}$, although in this latter case the spectrum includes emission lines and contamination from the background source that affects the SSP fitting. These results are indicative that the total density profile is approximately isothermal (Auger et al. 2010).

\section{OTHER GALAXIES IN THE MUSE FIELD}

We have used an analytical Sérsic fitting approach to remove the lens contribution from the spectra of all other galaxies in the field of view.

The way to model the spectrophotometric properties of the lens is using the two-dimensional photometric decomposition code GASP2D (Méndez-Abreu et al. 2008, 2014). It allows to fit a Sérsic model to the galaxy surface brightness distribution, thus deriving the main structural parameters of the galaxy, i.e., effective surface brightness $\left(\mu_{e}\right)$, effective radius $\left(r_{\text {eff }}\right)$, Sérsic index $(n)$, ellipticity $(\epsilon)$, and position angle $(P A)$. GASP2D uses an iterative LevenbergMarquardt algorithm to find the solution that minimise the $\chi^{2}$. At each iteration, the galaxy model is convolved with the image Point Spread Function (PSF) before computing the $\chi^{2}$. In our case we consider the PSF to be a circular Moffat with Full Width at Half Maximum FWHM $=0.7$ arcsec and shape parameter $\beta=1.95$. Similarly as with the isophotal fitting, each wavelength slice of the datacube is treated as an independent galaxy image, and the best fit Sérsic parameters are derived. The averaged Sérsic parameters over the whole wavelength range of the model are: $r_{e} f f=8.8^{\prime \prime}(9.30 \mathrm{kpc})$, $n=5.2, \epsilon=0.1$, and $P A=51 \mathrm{deg}(\mathrm{N} \rightarrow \mathrm{E})$. It is worth noting that a test with a Sérsic+Exponential model was also performed. However, it did not improve the single Sérsic fit and we use the latter to avoid over-fitting.

Once the model is subtracted from the observed datacube, we have found 15 bright sources within the FoV, besides the lens, the source, and the merger. Table 1 lists their coordinates and spectroscopic redshifts of all objects detected in the FoV. To investigate the nature of their emission, we have extracted $1^{\prime \prime}$ diameter circular aperture spectra at those positions in both the observed and the lens model datacubes and studied the residual spectra. We have been able to characterize 14 of these 15 sources, and details are given below.

Two sources, labeled $K$ and $L$ in Figure 1, show only 4 strong ionized gas emission lines corresponding to starforming galaxies at redshift 0.09 , as shown in Figure 8. In this Figure we also present $4^{\prime \prime} \times 4^{\prime \prime}$ cutouts of the $\mathrm{H} \alpha 2 \mathrm{D}$ emission line map. $\mathrm{H} \alpha$ is the strongest line in the spectrum of $K$, which also shows $\mathrm{H} \beta$, [OIII] $\lambda 4959$, and [OIII] $\lambda 5007$. Galaxy $L$ has the same 4 lines but in this case [OIII] $\lambda 5007$ is stronger than $\mathrm{H} \alpha$. In the blue end of the spectrum there are indications of $\mathrm{H} \gamma$ in both spectra, but only the red wing is visible. [N II] $\lambda \lambda 6548,6583$ doublet is not present in any of the two galaxies, which avoids estimating oxygen abundance through typical empirical calibrations. However, in both spectra [OIII] $\lambda 5007$ is stronger than $\mathrm{H} \beta$ indicating 

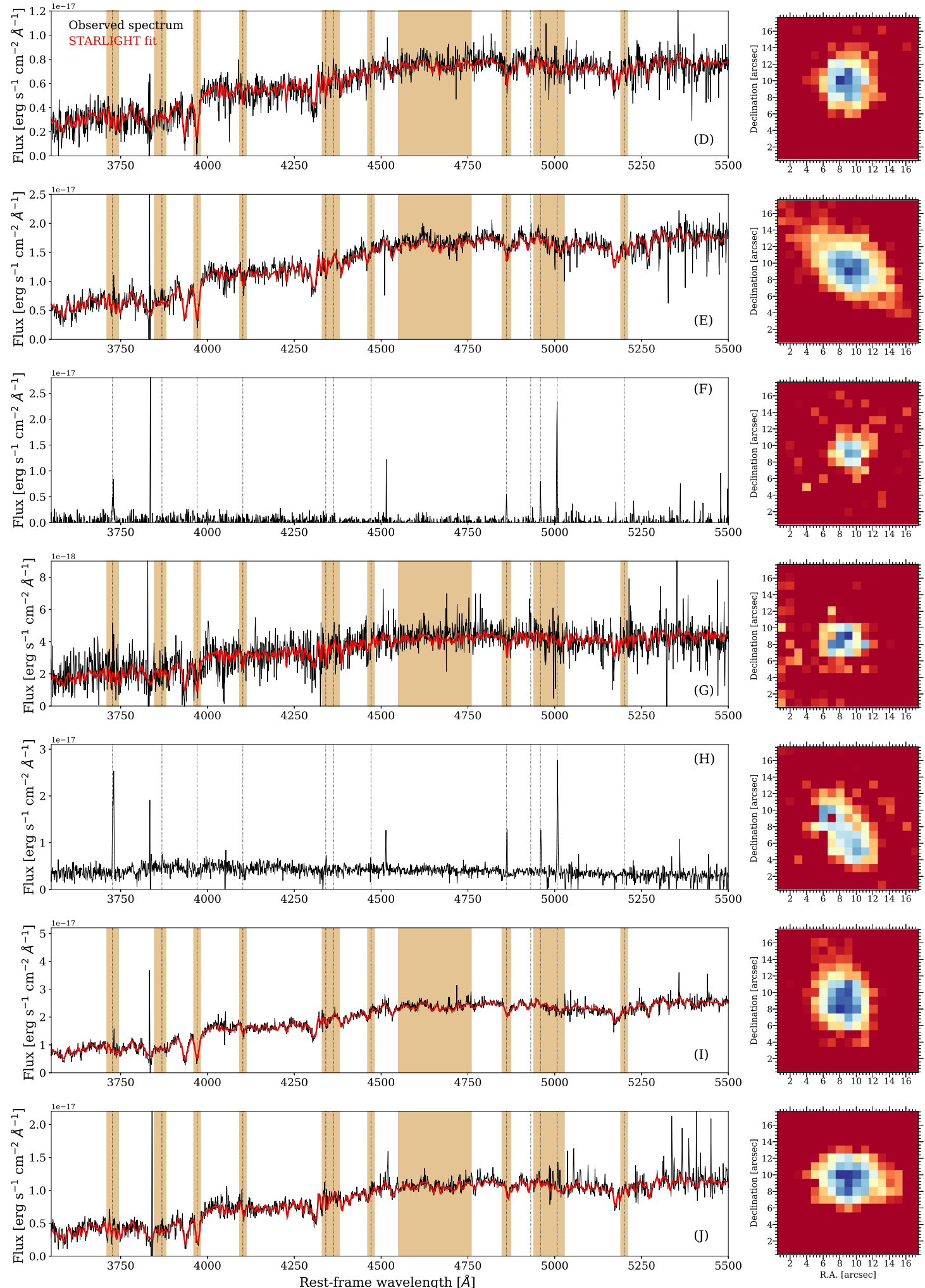

Figure 9. Spectra of background galaxies $D$ to $J$, all at redshifts $z \sim 0.45$. We show the observed spectrum in black and the best STARLIGHT fit in red. Brown regions are masked in the STARLIGHT fit, while the wavelengths of the most prominent emission lines are marked with vertical dotted lines. On the right, we show $4^{\prime \prime} \times 4^{\prime \prime}$ cutouts of the $\mathrm{S} / \mathrm{N} 2 \mathrm{D}$ map. For galaxies $F$ and $H$ we show the $[\mathrm{O}$ III] $\lambda 5007$ emission map instead. 

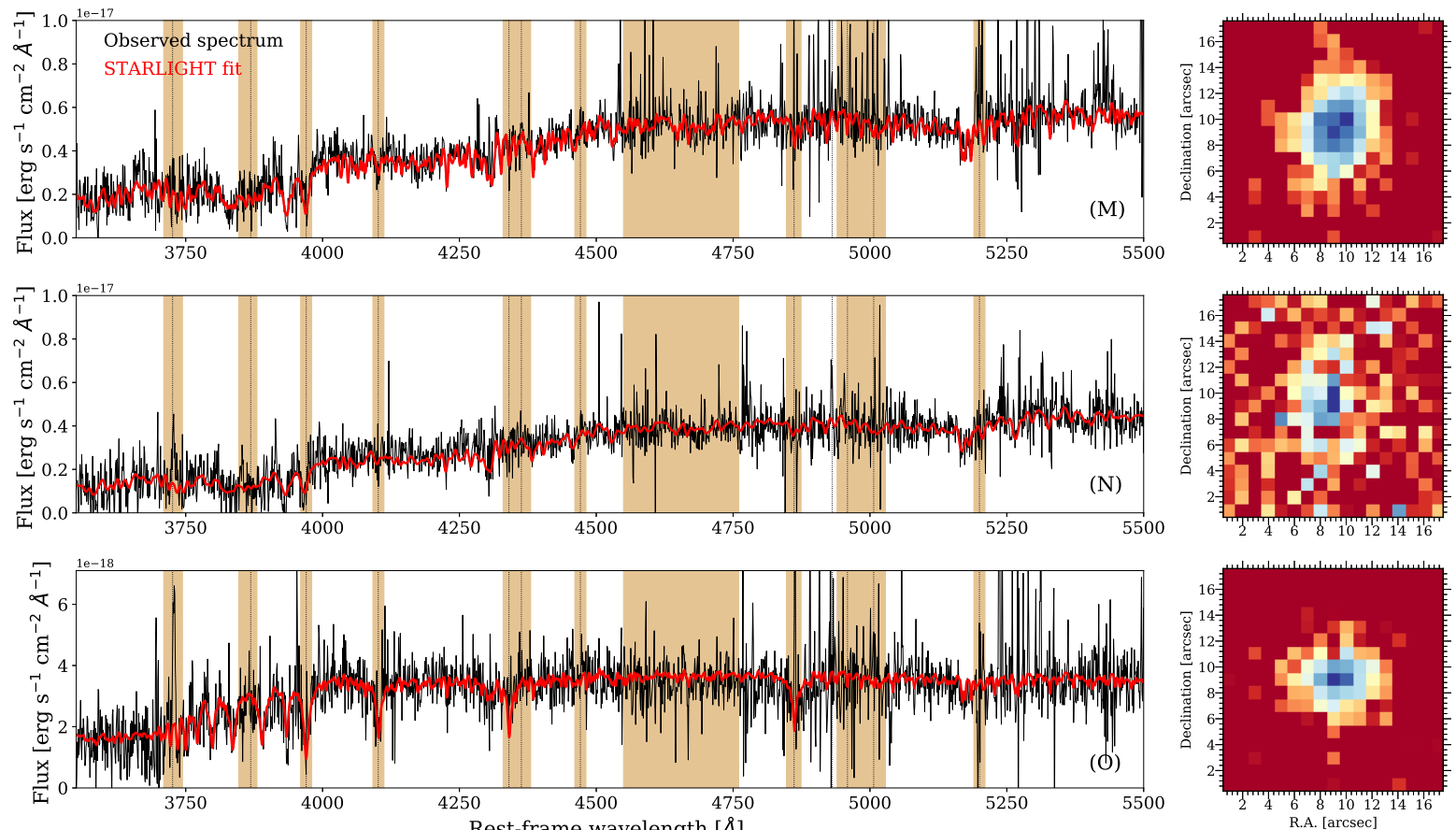

Figure 10. Similar to Figure 9 for galaxies $M, N$, and $O$ detected at redshifts $z \sim 0.59$.
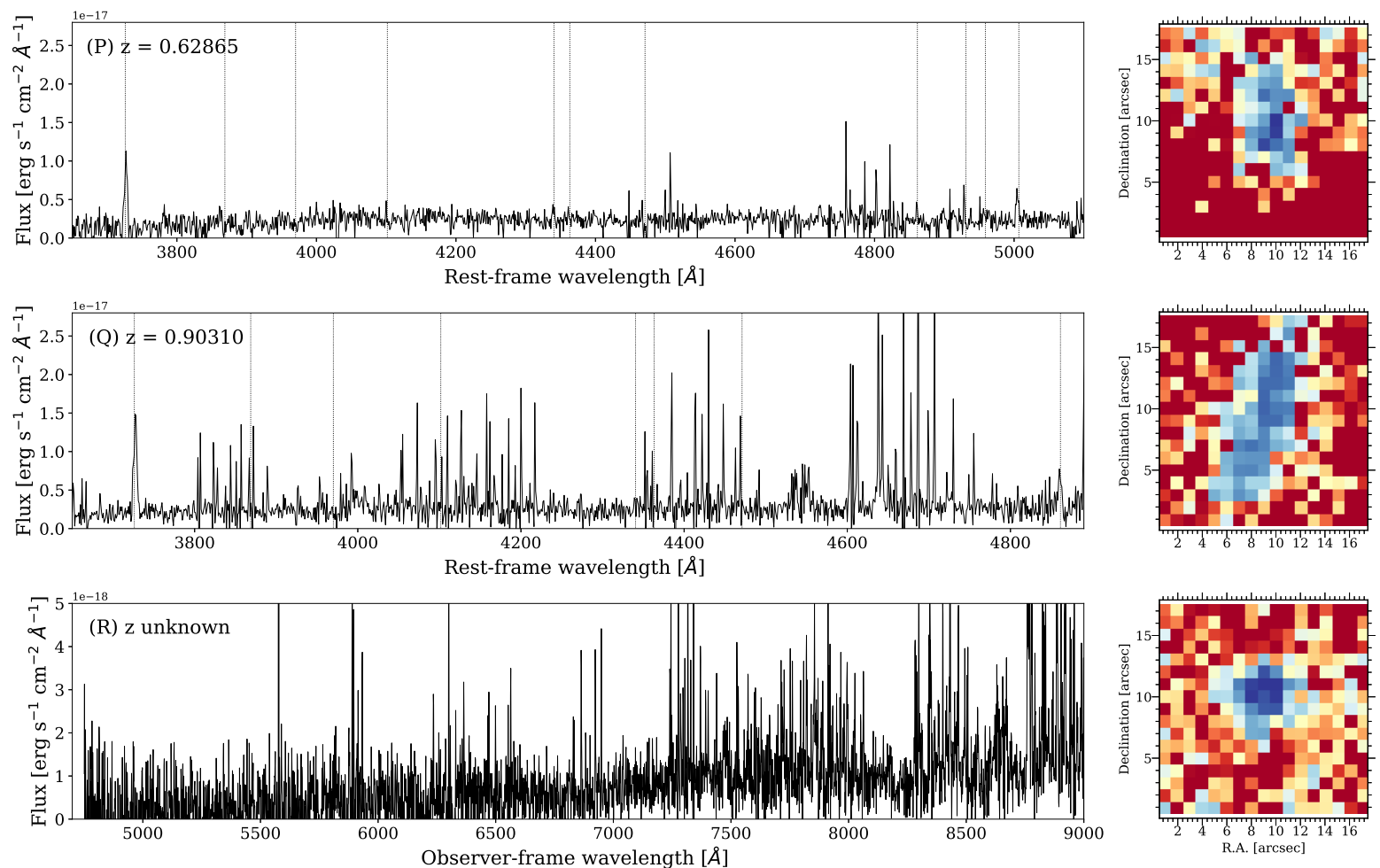

Figure 11. Similar to Figure 9 for galaxies $P$ and $Q$ detected at redshifts $z \sim 0.62$ and 0.90 , respectively, and object $R$ at unknown redshift. 
low-metallicity. No STARLIGHT fit was performed due to the faint stellar continuum, but fluxes of all prominent emission lines are reported in Table 3.

Further than $K$ and $L$, we find the lensed source galaxy at $\mathrm{z}=0.1915$, and following that we detected 7 different sources at redshifts around 0.45. They are all marked in Figure 1 with yellow circles, and labeled with letters $D$ to $J$. In Figure 9 we present all their spectra extracted from a 1 arcsec diameter aperture, together with $4^{\prime \prime} \times 4^{\prime \prime}$ cutouts of the $\mathrm{S} / \mathrm{N}$ of the spectra as measured in the observed-frame $50 \AA$ window centered at $6000 \AA$. Five objects had a bright red continuum and no emission lines representative of passive galaxies dominated by old stellar populations. We fit these spectra with STARLIGHT and provide their properties in Table 4 . Objects $F$ and $H$ have significantly fainter continua and present a few strong emission lines. For this reason, in Figure 9 we show the 2D [O III] $\lambda 5007$ map instead of a S/N $4^{\prime \prime} \times 4^{\prime \prime}$ cutout. In both galaxies, the spectrum does not cover the wavelength region corresponding to $\mathrm{H} \alpha$ in the rest-frame, and [O III] $\lambda 5007$ is the strongest emission line. Again, this line is stronger than $\mathrm{H} \beta$ indicating metalpoor nature.

The next set of galaxies, $M, N$, and $O$ in Figure 1, are at redshift $\sim 0.59$. All their spectra, shown in Figure 10, are compatible to passive red galaxies at $z \sim 0.59$. We were able to fit STARLIGHT to the three spectra, and their properties are listed in Table 4.

Finally, in Figure 11 we present the spectra and cutouts of the other 3 galaxies found in the FoV. The $\mathrm{S} / \mathrm{N}$ of these spectra are too low for fitting SSP with STARLIGHT, but we are able to determine the redshift from their emission lines in two cases: object $P$ is at $z=0.6286$ according to the [OII] $\lambda \lambda 3727,29$ and [OIII] $\lambda 5007$ lines, and object $Q$ is at 0.9031 from [OII] $\lambda \lambda 3727,29$ and $\mathrm{H} \beta$ lines. We have not been able to find good matches for object $R$, although we are convinced of the detection from its red continuum and the blob in the S/N map. New, deeper spectroscopic observation is needed to provide further information for object $R$.

\section{SUMMARY AND DISCUSSION}

We reported the discovery of a strong lensed galaxy at $z=0.1915$ by the lens 2MASX J04035024-0239275 at $z=0.0661$ in IFS observations from MUSE at the $8.2 \mathrm{~m}$ VLT of Cerro Paranal.

Two images from the source are identified and, after a careful analysis, confirmed as lensed images coming from the same source. We modeled the lens with a SIE mass profile and a Sérsic background source, finding an Einstein radius of $1.45 \pm 0.04{ }^{\prime \prime}(1.9 \mathrm{kpc})$, consistent with an isothermal density profile.

Independent of our discovery, a recent archival search of public MUSE data by Collier et al. (2018) also identified this galaxy as a strong gravitational lens. While they predict the dark matter fraction from cosmological hydrodynamical simulations, we derive our stellar mass estimate from the SSP synthesis with STARLIGHT to the MUSE data integrated within the Einstein radius. In both cases, we find a consistent fraction of dark matter within the Einstein radius ( $\sim 18 \%)$. However, the main difference between both works is the approach used to estimate the average age of the stel-

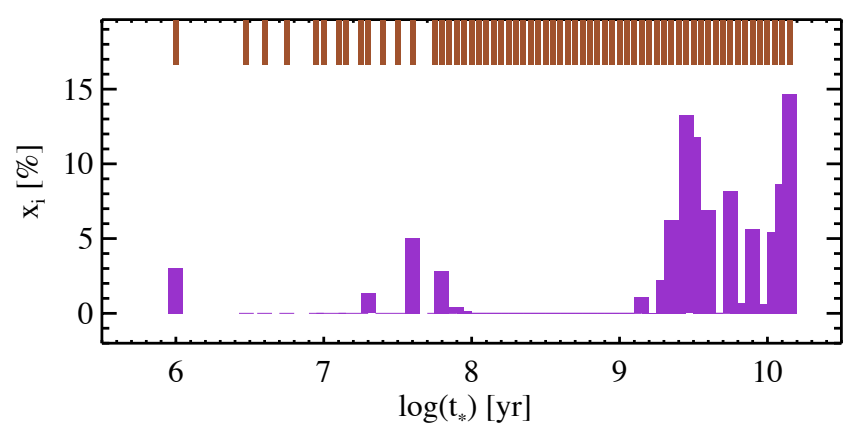

Figure 12. Star formation history of the spectrum extracted from the observed MUSE cube with an aperture of the Einstein radius (1.45 arcsec). Vertical brown lines on top correspond to the ages of the base SSPs used in the STARLIGHT fit. The light-weighted average age is $2.6 \mathrm{Gyr}$. We recover a small fraction of $<1 \mathrm{Gyr}$ populations, and two clear peaks around 2.8 and $12.5 \mathrm{Gyr}$.

lar populations in the lens galaxy. This may have significant consequences when using such parameters to infer the initial mass function (IMF) excess parameter (alpha). Indeed, Collier et al. claim a lightweight IMF rather than a standard Salpeter value for the lens galaxy.

Collier et al. estimate a reference mass-to-light ratio, $(\mathrm{M} / \mathrm{L})_{\text {ref }}$, for the IMF excess parameter by fitting a singleburst model to a spectrum extracted with an aperture of the Einstein radius. Following this approach, they are able to recover the dominant population which has a stellar age of $12 \mathrm{Gyr}$ and a metallicity $1.5 \mathrm{Z} \odot$. Just considering this single population, they are making the strong/uncertain assumptions that: (1) all stars in the galaxy have the same age, metallicity, and were created at the same time; (2) the M/L is constant through the extension of the Einstein radius; and (3) the IMF is constant through the same extension. All these assumptions are at odds with current understanding of galaxy formation and evolution from spatially resolved analyses of SPs where each population has its own specific M/L. In addition, these assumptions are clearly not in agreement with the current view of elliptical galaxies (e.g. see Fig. 5 in Sánchez et al. 2018, and Fig. 8 in González Delgado et al. 2015).

A more detailed approach would consist on performing SSP synthesis to infer the full star formation history. The analysis with STARLIGHT on the spectrum of an aperture of the Einstein radius $\left(1.45^{\prime \prime}\right)$ provides an average age of 2.6 Gyr and metallicity around solar (See Figure 12). We recover SPs of old ages (>10 dex) but a whole range of younger populations, in particular two peaks at 2.8 and $12.5 \mathrm{Gyr}$ (9.4 and $10.1 \mathrm{dex}$ ). As a comparison, Figure 8 in Gallazzi et al. (2005) shows the distribution of age and metallicity as a function of stellar mass for high signal-to-noise SDSS galaxies. For the stellar mass of the lens galaxy, $10.94 \mathrm{dex}$, our estimates of age and metallicity are within 1 sigma of the SDSS distribution, while those by Collier et al. are outliers. In addition, Portinari et al. (2004) in their Figure 2 show that for stellar ages of $12 \mathrm{Gyr}$ the $(\mathrm{M} / \mathrm{L})_{\text {ref }}$ is around a factor 2 larger than for populations of $\sim 3 \mathrm{Gyr}$ ages. Therefore, our extracted values would provide a much lower $(\mathrm{M} / \mathrm{L})_{\text {ref }}$ and an $\alpha \gtrsim 1.5$ totally compatible with a Salpeter IMF.

Finally, we note that to discriminate between different 
IMFs is unfeasible considering (among other reasons): (i) the spectral range covered by the current dataset, laking the blue part of the spectrum, in particular de D4000 region; (ii) our current uncertainties in the SSPs libraries, which make it difficult to measure the $\mathrm{M} / \mathrm{L}$ at the precision required; (iii) not having a good way to measure the [alpha/Fe] gradient which, by itself, could mimic the observed discrepancy; and (iv) the known uncertainties in the fitting procedures. Finally, we stress that putting constraints on the IMF was not the main goal of this work, and we refer to state-of-the-art IMF derivations, such as those from Martín-Navarro et al. (2015) or by the Atlas3D group (Cappellari et al. 2012), to outline the complexity of the matter.

Fifteen other galaxies have been detected in the 1 $\operatorname{arcmin}^{2} \mathrm{FoV}$, in addition to the identification of a possible merger with the lensing galaxy. None of these background sources are strongly lensed. We provided spectroscopic redshifts and stellar and ionized gas properties for 14 of these background galaxies. Object $R$ shows a red continuum, but we were unable to get reliable constrains on its redshift. Deeper observations are needed for such constrains.

In this work we have been able to put constraints on the dark matter content within the Einstein radius of a nearby elliptical galaxy acting as a lens for a background galaxy, and present an analysis in great detail. Looking to the future, the advent of large surveys such as the Large Synoptic Survey Telescope (LSST) will be able to discover a significant number of new strong-lensed systems, and we demonstrated how wide-field Integral field spectroscopy offers an excellent approach to study them and to precisely model its effects.

\section{ACKNOWLEDGEMENTS}

We are greatly thankful to Michael Wood-Vasey, Rachel Mandelbaum, Santiago González-Gaitán, Lindsay Oldham, Andrew Zentner, and Jeff Newman, for very valuable input on the early stages of this project. L.G. was supported in part by the US National Science Foundation under Grant AST-1311862. TEC is funded by a Dennis Sciama Fellowship from the University of Portsmouth. Based on observations made with ESO Telescopes at the La Silla Paranal Observatory under program 98.D-0115(A). Llenya d'alzina, vi de sarment, oli d'oliva, pa de forment.

\section{REFERENCES}

Auger M. W., Treu T., Bolton A. S., Gavazzi R., Koopmans L. V. E., Marshall P. J., Moustakas L. A., Burles S., 2010, ApJ, 724, 511

Bacon R., et al., 2010, in Ground-based and Airborne Instrumentation for Astronomy III. p. 773508, doi:10.1117/12.856027

Bekki K., Couch W. J., Shioya Y., 2002, ApJ, 577, 651

Belfiore F., Maiolino R., Bothwell M., 2016, MNRAS, 455, 1218 Bertin G., et al., 1994, A\&A, 292, 381

Binette L., Magris C. G., Stasińska G., Bruzual A. G., 1994, A\&A, 292, 13

Blakeslee J. P., et al., 2004, ApJ, 602, L9

Bolton A. S., Burles S., Koopmans L. V. E., Treu T., Moustakas L. A., 2006, ApJ, 638, 703

Bonvin V., et al., 2017, MNRAS, 465, 4914

Bundy K., et al., 2015, ApJ, 798, 7
Cappellari M., et al., 2012, Nature, 484, 485

Cappellari M., et al., 2013, MNRAS, 432, 1709

Cappellari M., et al., 2015, ApJ, 804, L21

Cardelli J. A., Clayton G. C., Mathis J. S., 1989, ApJ, 345, 245

Charbonnel C., Meynet G., Maeder A., Schaller G., Schaerer D., 1993, A\&AS, 101, 415

Cheung E., et al., 2016, Nature, 533, 504

Cid Fernandes R., Mateus A., Sodré L., Stasińska G., Gomes J. M., 2005, MNRAS, 358, 363

Collett T. E., Auger M. W., 2014, MNRAS, 443, 969

Collier W. P., Smith R. J., Lucey J. R., 2018, preprint, (arXiv:1803.07082)

Einstein A., 1915, Sitzungsberichte der Königlich Preußischen Akademie der Wissenschaften (Berlin), Seite 844-847.,

Falcón-Barroso J., Sánchez-Blázquez P., Vazdekis A., Ricciardelli E., Cardiel N., Cenarro A. J., Gorgas J., Peletier R. F., 2011, A\&A, 532, A95

Galbany L., et al., 2014, A\&A, 572, A38

Galbany L., et al., 2016a, MNRAS, 455, 4087

Galbany L., et al., 2016b, A\&A, 591, A48

Galbany L., et al., 2018, ApJ, 855, 107

Gallazzi A., Charlot S., Brinchmann J., White S. D. M., Tremonti C. A., 2005, MNRAS, 362, 41

García-Lorenzo B., Sánchez S. F., Mediavilla E., GonzálezSerrano J. I., Christensen L., 2005, ApJ, 621, 146

Gavazzi R., Marshall P. J., Treu T., Sonnenfeld A., 2014, ApJ, 785,144

Girardi L., Bressan A., Bertelli G., Chiosi C., 2000, A\&AS, 141, 371

Gomes J. M., et al., 2016a, A\&A, 585, A92

Gomes J. M., et al., 2016b, A\&A, 588, A68

González Delgado R. M., Cerviño M., Martins L. P., Leitherer C., Hauschildt P. H., 2005, MNRAS, 357, 945

González Delgado R. M., et al., 2015, A\&A, 581, A103

Jones D. H., et al., 2009, MNRAS, 399, 683

Kauffmann G., et al., 2003, MNRAS, 346, 1055

Kehrig C., et al., 2012, A\&A, 540, A11

Kennicutt Jr. R. C., 1998, ARA\&A, 36, 189

Kewley L. J., Dopita M. A., Sutherland R. S., Heisler C. A., Trevena J., 2001, ApJ, 556, 121

Krühler T., Kuncarayakti H., Schady P., Anderson J. P., Galbany L., Gensior J., 2017, A\&A, 602, A85

Lanusse F., Ma Q., Li N., Collett T. E., Li C.-L., Ravanbakhsh S., Mandelbaum R., Póczos B., 2018, MNRAS, 473, 3895

Marino R. A., et al., 2013, A\&A, 559, A114

Martín-Navarro I., La Barbera F., Vazdekis A., Falcón-Barroso J., Ferreras I., 2015, MNRAS, 447, 1033

Méndez-Abreu J., Aguerri J. A. L., Corsini E. M., Simonneau E., 2008, A\&A, 478, 353

Méndez-Abreu J., Debattista V. P., Corsini E. M., Aguerri J. A. L., 2014, A\&A, 572, A25

Narayan R., Bartelmann M., 1996, ArXiv Astrophysics e-prints,

Newman A. B., Smith R. J., Conroy C., Villaume A., van Dokkum P., 2017, ApJ, 845, 157

Papaderos P., et al., 2013, preprint, (arXiv:1306.2338)

Planck Collaboration et al., 2016, A\&A, 594, A13

Portinari L., Sommer-Larsen J., Tantalo R., 2004, MNRAS, 347, 691

Salpeter E. E., 1955, ApJ, 121, 161

Sánchez S. F., et al., 2016, Rev. Mex. Astron. Astrofis., 52, 21

Sanchez S. F., et al., 2017, preprint, (arXiv:1709.05438)

Sánchez S. F., et al., 2018, Rev. Mex. Astron. Astrofis., 54, 217

Sarzi M., et al., 2010, MNRAS, 402, 2187

Schaerer D., Meynet G., Maeder A., Schaller G., 1993a, A\&AS, 98,523

Schaerer D., Charbonnel C., Meynet G., Maeder A., Schaller G., 1993b, A\&AS, 102, 339 


\section{Galbany et al.}

Schaller G., Schaerer D., Meynet G., Maeder A., 1992, A\&AS, 96,269

Schlafly E. F., Finkbeiner D. P., 2011, ApJ, 737, 103

Singh R., et al., 2013, A\&A, 558, A43

Smith R. J., 2017, MNRAS, 464, L46

Smith R. J., Blakeslee J. P., Lucey J. R., Tonry J., 2005, ApJ, 625, L103

Smith R. J., Lucey J. R., Conroy C., 2015, MNRAS, 449, 3441

Stasińska G., et al., 2008, MNRAS, 391, L29

Talbot M. S., et al., 2018, MNRAS,

Treu T., 2010, ARA\&A, 48, 87

Vazdekis A., Sánchez-Blázquez P., Falcón-Barroso J., Cenarro A. J., Beasley M. A., Cardiel N., Gorgas J., Peletier R. F., 2010, MNRAS, 404, 1639

Walker E. S., et al., 2013, The Astronomer's Telegram, 5567

Zwicky F., 1937, Physical Review, 51, 290 
Table 3. Extinction corrected fluxes of the most prominent gas phase emission lines. All fluxes in units of $10^{-18} \mathrm{erg} \mathrm{s}^{-1} \mathrm{~cm}^{-2} \AA^{-1}$.

\begin{tabular}{|c|c|c|c|c|c|c|c|c|c|c|}
\hline Galaxy & {$[\mathrm{O} \mathrm{II}] \lambda \lambda 3727,29$} & $\mathrm{H} \delta$ & $\mathrm{H} \gamma$ & $\mathrm{H} \beta$ & [O III] $\lambda 4959$ & {$[\mathrm{O} \mathrm{III}] \lambda 5007$} & {$[\mathrm{~N} \mathrm{II}] \lambda 6548$} & $\mathrm{H} \alpha$ & {$[\mathrm{N} \mathrm{II}] \quad \lambda 6583$} & [S II] $\lambda 671$ \\
\hline $\mathrm{D}$ & - & - & - & - & - & - & - & - & - & - \\
\hline $\mathrm{E}$ & - & - & - & - & - & - & - & - & - & - \\
\hline $\mathrm{F}$ & $26.89(7.67)$ & - & - & $12.19(5.94)$ & $22.04(9.27)$ & $76.08(27.40)$ & - & - & - & - \\
\hline G & - & - & - & - & - & - & - & - & - & - \\
\hline $\mathrm{H}$ & $96.54(21.82)$ & - & - & $28.70(11.09)$ & $25.91(9.88)$ & $71.83(24.28)$ & - & - & - & - \\
\hline I & - & - & - & - & - & - & - & - & - & - \\
\hline $\mathrm{J}$ & - & - & - & - & - & - & - & - & - & - \\
\hline $\mathrm{K}$ & - & - & - & $57.56(20.42)$ & $29.46(11.50)$ & $84.40(31.79)$ & - & $164.62(61.47)$ & - & $164.62(61$. \\
\hline $\mathrm{L}$ & - & - & - & $236.77(86.09)$ & $416.37(154.50)$ & $1256.91(472.34)$ & - & $677.17(251.72)$ & - & $677.17(251$. \\
\hline $\mathrm{M}$ & - & - & - & - & - & - & - & - & - & - \\
\hline $\mathrm{N}$ & $26.39(3.69)$ & - & - & - & - & - & - & - & - & - \\
\hline $\mathrm{O}$ & $24.03(4.67)$ & - & - & - & - & - & - & - & - & - \\
\hline $\mathrm{P}$ & $47.30(9.43)$ & - & $9.79(2.38)$ & - & - & $16.58(3.66)$ & - & - & - & - \\
\hline $\mathrm{Q}$ & $59.05(12.89)$ & - & - & $20.78(4.70)$ & - & - & - & - & - & - \\
\hline
\end{tabular}

Table 4. Properties extracted from aperture spectra of all sources in the residual cube after subtracting the lens model, except for the lens which was extracted in the observed cube.

\begin{tabular}{|c|c|c|c|c|c|c|c|}
\hline Galaxy & $\begin{array}{l}\mathrm{A}_{V}^{\text {gas }} \\
{[\mathrm{mag}]}\end{array}$ & $\begin{array}{c}12+\log _{10}(\mathrm{O} / \mathrm{H}) \\
{[\mathrm{dex}]}\end{array}$ & 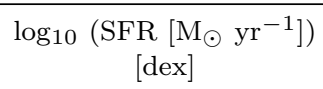 & $\begin{array}{c}\log _{10}\left(\mathrm{M}\left[\mathrm{M}_{\odot}\right]\right) \\
{[\mathrm{dex}]}\end{array}$ & $\begin{array}{c}\mathrm{A}_{V}^{*} \\
{[\mathrm{mag}]}\end{array}$ & $\begin{array}{c}<\log _{10}\left(\mathrm{t}_{*}[\mathrm{yr}]\right)> \\
{[\mathrm{dex}]}\end{array}$ & $\begin{array}{c}<\log _{10} Z_{*}> \\
{[\mathrm{dex}]}\end{array}$ \\
\hline D & - & - & - & 10.63 & 0.00 & $9.73(0.35)$ & $0.027(0.007)$ \\
\hline $\mathrm{E}$ & - & - & - & 11.11 & 0.17 & $9.84(0.33)$ & $0.023(0.014)$ \\
\hline $\mathrm{F}$ & - & - & - & - & - & - & - \\
\hline G & - & - & - & 10.27 & 0.10 & $9.53(0.52)$ & $0.024(0.014)$ \\
\hline $\mathrm{H}$ & - & - & - & - & - & - & - \\
\hline I & - & - & - & 11.18 & 0.00 & $9.64(0.33)$ & $0.032(0.000)$ \\
\hline $\mathrm{J}$ & - & - & - & 10.75 & 0.00 & $9.64(0.38)$ & $0.031(0.003)$ \\
\hline K & $0.31(0.03)$ & - & 0.031526 & - & - & - & - \\
\hline $\mathrm{L}$ & $0.05(0.02)$ & - & 0.129686 & - & - & - & - \\
\hline M & - & - & - & 10.89 & 0.00 & $9.85(0.35)$ & $0.032(0.000)$ \\
\hline $\mathrm{N}$ & - & - & - & 11.06 & 0.09 & $10.12(0.08)$ & $0.019(0.000)$ \\
\hline $\mathrm{O}$ & - & - & - & 9.91 & 0.05 & $8.98(0.33)$ & $0.023(0.008)$ \\
\hline $\mathrm{P}$ & - & - & - & - & - & - & - \\
\hline $\mathrm{Q}$ & - & - & - & - & - & - & - \\
\hline $\mathrm{R}$ & - & - & - & - & - & - & - \\
\hline
\end{tabular}

